\title{
Dimer-resonating valence bond state of the four-leg Heisenberg ladder: Interference among resonances
}

\author{
M. Roncaglia and G. Sierra \\ Instituto de Matemáticas y Física Fundamental, C.S.I.C., Madrid, Spain \\ M. A. Martin-Delgado \\ Departamento de Física Teórica, Universidad Complutense, Madrid, Spain
}

(Received 6 July 1999)

\begin{abstract}
We study the ground state of the four-leg spin ladder using a dimer-resonating valence bond (RVB) ansatz and the Lanczos method. Besides the well-known resonance mechanism between valence bond configurations we find interference effects among nearby resonances. These effects were missed by standard factorizing approaches to the RVB states. [S0163-1829(99)08441-6]
\end{abstract}

\section{INTRODUCTION}

Doped and undoped ladders have focused a lot of attention in recent years due to the existence of materials with that structure; some of them are close relatives of the high- $T_{c}$ oxides as the series $\mathrm{Sr}_{2 n_{l}-2} \mathrm{Cu}_{2 n_{l}} \mathrm{O}_{4 n_{l}-2}$ where $n_{l}$ is the number of legs forming the ladder. ${ }^{1}$ The undoped Heisenberg spin ladders with $n_{l}$ even are known to be spin liquids with a spin gap and exponential decaying correlation functions. ${ }^{2}$ The ground state (GS) of these low dimensional systems is given by a short-range resonating valence bond (RVB) ansatz where the topological spin defects are confined. ${ }^{3}$ The RVB picture is supported by mean field, ${ }^{5}$ density matrix renormalization group (DMRG) ${ }^{3,4}$ quantum Monte Carlo (QMC), ${ }^{6}$ and Lanczos $^{7}$ results concerning ladders with $n_{l}$ =2,4 legs and variational Ansätze (RVA) for the 2-leg ladder. ${ }^{8,9}$ The purpose of this paper is to apply the RVA method to the four-leg spin ladder with the aim of studying in more detail the structure of the short-range RVB state. In the two-leg ladder case the basic mechanism that lowers the GS energy is the resonance between two nearest-neighbor valence bonds. ${ }^{10}$ The simplest short-range RVB Ansatz is given by a dimer-RVB state with a single variational parameter $u$, which gives the amplitude of the resonance. ${ }^{8,9}$ See Ref. 11 for a transfer matrix approach to dimer-RVB states.

\section{RVA APPROACH TO THE FOUR-LEG HEISENBERG LADDER}

In the four-leg ladder case we shall study a dimer-RVB Ansatz where the resonance may occur among any possible pair of nearest-neigbor parallel bonds. The phenomenon we shall investigate in this paper is the "interference" between couples of resonating bonds. We mean by interference the influence that a pair of resonating bonds exerts on another pair of nearby resonating bonds. In the standard RVB Ansatz of Liang et al. ${ }^{12}$ the RVB amplitudes have a factorized form that cannot describe this interference effect.

The Hamiltonian of the four-leg spin ladder is given by

$$
\begin{aligned}
H= & J \sum_{a=1}^{4} \sum_{n=1}^{N-1} \mathbf{S}_{a}(n) \cdot \mathbf{S}_{a}(n+1)+\sum_{n=1}^{N}\left[J ^ { \prime } \left\{\mathbf{S}_{1}(n) \cdot \mathbf{S}_{2}(n)\right.\right. \\
& \left.\left.+\mathbf{S}_{3}(n) \cdot \mathbf{S}_{4}(n)\right\}+J^{\prime \prime} \mathbf{S}_{2}(n) \cdot \mathbf{S}_{3}(n)+J^{\prime \prime \prime} \mathbf{S}_{1}(n) \cdot \mathbf{S}_{4}(n)\right]
\end{aligned}
$$

where $\mathbf{S}_{a}(n)$ is the spin $1 / 2$ operator at the $a=1, \ldots, 4$ leg and $n=1, \ldots, N$ rung. We shall consider the cases of periodic or closed boundary conditions (BC) along the rungs, i.e., $J^{\prime}=J^{\prime \prime}=J^{\prime \prime \prime}$ and open BC's along the rungs, i.e., $J^{\prime}$ $=J^{\prime \prime}, J^{\prime \prime \prime}=0$. Setting $J^{\prime \prime}=J^{\prime \prime \prime}=0$ we recover two decoupled two-leg ladder Hamiltonians. If $J=0$, the exact GS of Eq. (1) is given by the coherent superposition of the GS of every rung which can be written as

$$
\begin{gathered}
\mid \text { rung }\rangle=\overline{12} \overline{34}+u_{0} \overline{14} \overline{32,} \\
u_{0}= \begin{cases}1 & J^{\prime}=J^{\prime \prime}=J^{\prime \prime \prime} \\
0.366 & J^{\prime}=J^{\prime \prime}, J^{\prime \prime \prime}=0 \\
0 & J^{\prime \prime}=J^{\prime \prime \prime}=0,\end{cases}
\end{gathered}
$$

where $\overline{a b}=\left(|\uparrow\rangle_{a}|\downarrow\rangle_{b}-|\downarrow\rangle_{a}|\uparrow\rangle_{b}\right) / \sqrt{2}$ denotes the valence bond state between the sites on the legs $a$ and $b$ of the $n$th rung. In Figs. 1(a) and 1(b) we depict the valence bond states (2).

Switching on the intraleg coupling $J$ any pair of rung bonds will start to resonate with a pair of leg bonds as in Figs. 1(c)-1(f). There are four types of "elementary" resonances involving two consecutive rungs $n$ and $n+1$ and two legs $i$ and $j$, which we denote as (12), (34), (14), and (23). We associate an amplitude $u_{i j}$ to every one of these resonances. There is also a state with four leg bonds on two consecutive rungs, which we denote as (1234), and give it an amplitude $u_{1234}$ [see Fig. $1(\mathrm{~g})$ ]. Finally, we may have two resonances $(i j)$ and $(k l)$ sharing a common rung as in Figs. 1(h) and 1(i), which we denote as (12,34) and (14,23), and give them amplitudes $u_{12,34}=u_{34,12}$ and $u_{14,23}=u_{23,14}$, respectively. In this fashion we are able to retrieve a relevant small set of variational parameters out of the huge set of all resonating configurations. 


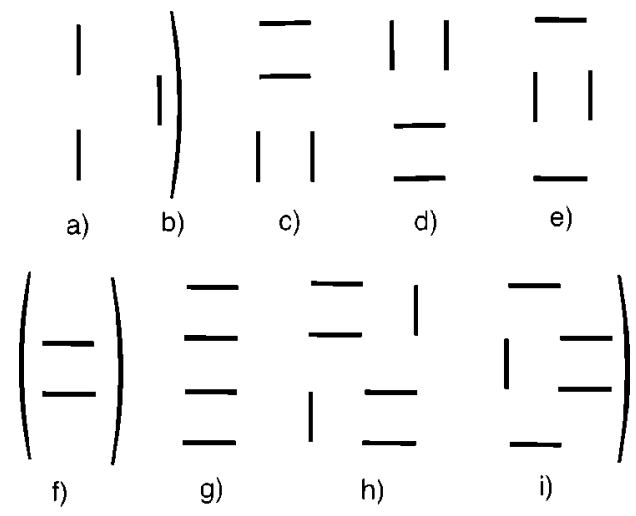

FIG. 1. Graphical representation of the local configurations that make up the dimer-RVB Ansatz for the four-leg ladder. Every line connecting two sites $a$ and $b$ corresponds to the valence bond state $\overline{a b}$ defined in the text. Site $a$ belongs to the even sublattice while site $b$ belongs to the odd one.

Let us suppose that a pair of resonating bonds is not influenced by its environment. This would imply the following factorization $u_{i j, k l}=u_{i j} u_{k l}$, which, as we shall see below, never happens. Figure 1 displays all the local configurations that should be combined in all possible manners to produce a dimer-RVB Ansatz. This seems to be a formidable problem if we try to solve it with standard combinatorial methods. However, as in the two-leg ladder case, ${ }^{9}$ the dimer-RVB state of the four-leg ladder can be generated by the set of recurrence relations (RR) given in Fig. 2.

Figure 3 shows a state generated by these RR's. One can characterize a dimer state with $N$ rungs by the collection of legs that one cuts between two consecutive rungs. If no legs are cut we write (0), cutting the legs $i$ and $j$ we write $(i j)$, and cutting four legs we write (1234). With these notations the state of Fig. 3 reads $(12)(0)(34)(12)(0)(23)$ and has an amplitude $u_{12} u_{12,34} u_{12} u_{23}$.

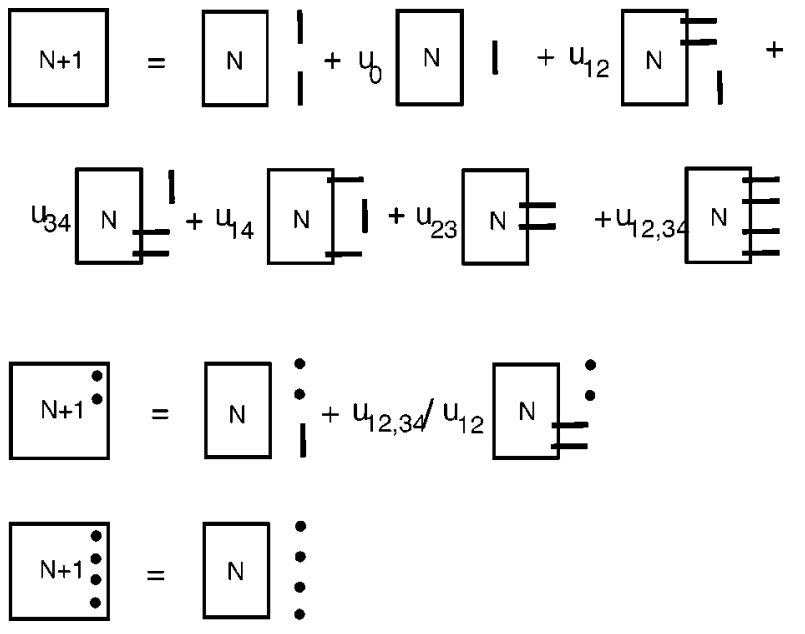

FIG. 2. Recurrence relations that generate iteratively the dimerRVB state of the four-leg ladder. The empty box represents the singlet state $|N\rangle$ of the ladder with $N$ rungs. A box with two dots on the legs $i$ and $j$ represents the state $\left|N, \sigma_{i}, \sigma_{j}\right\rangle$, where $\sigma_{i}$ and $\sigma_{j}$ are free spins that form valence bonds with nearest-neighbor spins located in the same legs. We give explicitly the RR of the state $\left|N, \sigma_{1}, \sigma_{2}\right\rangle$. The RR's of the other two-dotted states are similar. The last RR is that of the four-dotted state $\left|N, \sigma_{1}, \sigma_{2}, \sigma_{3}, \sigma_{4}\right\rangle$.

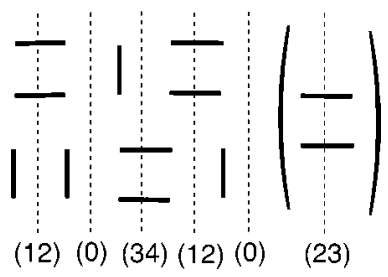

FIG. 3. A dimer state constructed with the RR's given in Fig. 2. The dotted lines represent the cuts described in the text.

It is important to realize that not all the states of the form $A_{12} A_{23} A_{34} \cdots A_{N-1, N}$ (where $A_{n, n+1}$ denotes the set of legs cut between the rungs $n$ and $n+1$ ) are allowed. For example, after the configuration (12) one can only have either (0) or (34), or after (1234) only (0) may follow. These selection rules are summarized in the graph of Fig. 4. The vertices of the graph denote the configurations $A=(0),(12)$, (34),(14),(23),(1234) while a link between the vertices $A$ and $A^{\prime}$ indicates that these two configurations may appear consecutively in an allowed dimer state. The amplitudes of the dimer states are associated to the links of the graph.

The RR's of Fig. 2 generate all the dimer states of a four-leg ladder with periodic BC's along the rungs, and their number grows exponentially with the number of legs. ${ }^{13}$ For the open BC's we should restrict ourselves to dimer states with no bonds of length greater than one. However, the strong coupling limit $J / J^{\prime} \ll 1$ forces us to include the valence bond $\overline{14}$ as in Eq. (2). So the distinction between closed and open ladders will only appear in the variational parameters. The existence of RR's to generate the GS Ansatz implies that the norm and expectation value of the Hamiltonian (1) also satisfies RR's, which can be iterated to give the energy of the Ansatz $\langle H\rangle_{N}$ for any number of rungs $N$. The set of variational parameters $u_{X}$ is obtained by minimization of $\langle H\rangle_{N}$. This method is similar to the matrix product Ansatz of Ref. 14 but differs in that the states kept are nonorthogonal as corresponds to a RVB Ansatz.

\section{RVA AND LANCZOS RESULTS}

We now present our set of results obtained with the recurrence variational Ansatz of the previous section and make also a Lanczos study of the four-leg ladder that we use as a

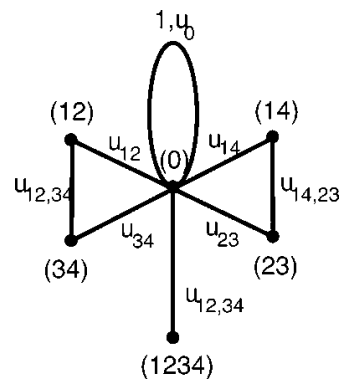

FIG. 4. Graph that encodes the dimer configurations of the fourleg ladder. The vertices are labeled by the legs cut between two consecutive rungs. A link between two vertices represents cuts that share a common rung. Every link is associated with a variational parameter of the RR's. The link connecting (0) to itself means that the middle rung between the two cuts is a singlet that may be either $\overline{12} \overline{34}$, with amplitude 1 or $\overline{14} \overline{23}$, with amplitude $u_{0}$. 


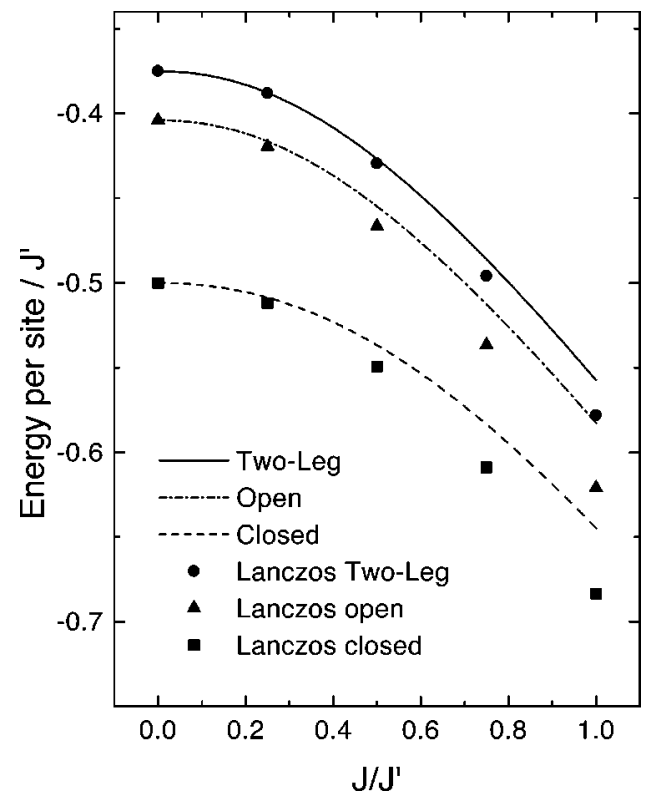

FIG. 5. GS energy per site in units of $J^{\prime}$ of the four-leg dimerRVB state with closed and open BC's and the two-leg ladder in the range $0<J / J^{\prime}<1$. We also plot the exact GS energies obtained by extrapolating Lanzcos results to the thermodynamic limit with ladders of sizes $4 \times n \quad(n=4,5,6,7)$.

test ground for the goodness of the RVA Ansatz.

In Fig. 5 we plot the GS energy per site obtained with our variational Ansatz and the Lanczos method in the range of couplings $0<J / J^{\prime}<1$. We also include for comparison the GS energy per site of the two-leg ladder. The GS energies are very close to the exact result in the strong coupling region $0<J / J^{\prime}<0.3$, but they get worse for larger couplings. This is natural since configurations with longer bonds are expected to become more important in the weak interleg coupling regime. The closed-rung ladder has a much lesser GS energy per site than the open one. This is mainly due to the resonance (2) between the two bonds along the rungs. The GS curves for open and closed ladders in Fig. 5 can be fitted with the formula,

$$
\begin{aligned}
& E_{0}(N) /\left(4 N J^{\prime}\right)=-e_{0}-e_{1}\left(J / J^{\prime}\right)^{2}-e_{2}\left(J / J^{\prime}\right)^{4}, \\
& \left(e_{0}, e_{1}, e_{2}\right)=\left\{\begin{array}{llll}
0.5, & 0.15, & -0.005, & \text { closed } \\
0.404, & 0.23, & -0.05 & \text { open }
\end{array}\right.
\end{aligned}
$$

where $e_{0}$ is the energy per site of a single rung. Equation (3) agrees with perturbation theory up to second order.

Let us consider now the behavior of the variational parameters. In the closed-rung case the choice of couplings $J^{\prime}=J^{\prime \prime}=J^{\prime \prime \prime}$ implies the existence of a rotational symmetry among the legs which leaves only four independent variational parameters given by

$$
u_{0}, \quad u_{12}=u_{i j}, \quad \mathrm{v}_{12,34}=\frac{u_{i j, k l}}{u_{i j} u_{k l}}, \quad u_{1234} .
$$

In Fig. 6 we plot these parameters in the domain 0 $<J / J^{\prime}<1$. Let us comment on these results.



FIG. 6. Variational parameters (4) of the closed BC dimer-RVB in the range $0<J / J^{\prime}<1$. We include for comparison the value of the variational parameter $u$ of the two-leg ladder. ${ }^{9}$

$u_{0}$ takes the constant value 1 , which coincides with the exact $J=0$ result (2). This implies the absence of interference between rung and leg resonance.

$u_{12}$ is greater than its two-leg analog $u .{ }^{9}$ For the isotropic case one gets $u_{12}=1.58$ while $u=1.18 .{ }^{9}$ Simple resonance is enhanced in the four-leg ladder.

$\mathrm{v}_{12,34}$ is almost constant and less than one indicating destructive interference between resonances shearing a common rung as in Fig. 1(h).

$u_{1234}$ displays an unexpected behavior since it first becomes negative for small values of $J / J^{\prime}$, reaches a minimum, and starts to grow becoming positive for $J / J^{\prime}>0.6$. This peculiar behavior of $u_{1234}$ is a sign of destructive interference between resonances sharing two rungs.

In the case of open ladders, $J^{\prime \prime}=J^{\prime}, J^{\prime \prime \prime}=0$, one is left with seven independent variational parameters given by

$$
u_{0}, \quad u_{12}=u_{34}, \quad u_{14}, \quad u_{23}, \quad \mathrm{v}_{i j, k l}=\frac{u_{i j, k l}}{u_{i j} u_{k l}}, \quad u_{1234} .
$$

In Fig. 7 we plot the values of these parameters in the range $0<J / J^{\prime}<1$. Some features that we encounter in Fig. 7 have already appeared in the periodic case.

$u_{0}$ stays almost constant with a value close to the exact $J=0$ result (2).

$u_{12}$ and $u_{14}$ are quite similar, but $u_{23}$ is much smaller. So bonds do not like to resonate in the middle of the ladder. This is due to loss of energy induced by the existence of the long bond $\overline{14}$.

$\mathbf{V}_{12,34}$ is lower than 1 , as in the periodic case, but $\mathbf{V}_{14,23}$ is much greater than 1 , which is again due to the smallness of $u_{23}$. For graphical purposes we plot in Fig. 7 the inverse of $\mathrm{v}_{14,23}$. 


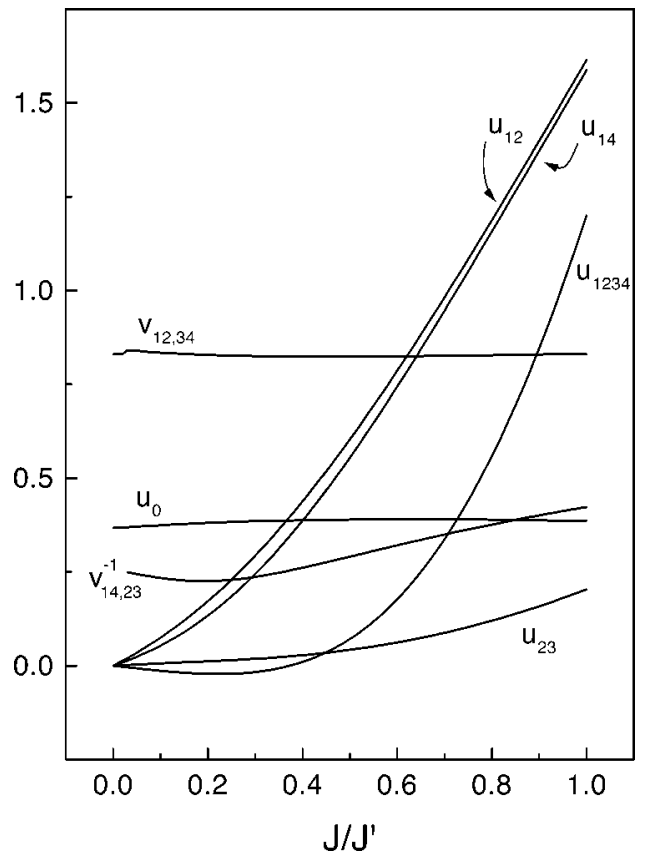

FIG. 7. Variational parameters (5) of the open BC dimer-RVB in the range $0<J / J^{\prime}<1$.

$u_{1234}$ is also suppressed but in a smaller amount than in the periodic ladder.

We have also computed the spin correlation length $\xi$ from the exponential decaying behavior of the spin-spin correlator. For the isotropic case we get $\xi=0.81$ for the closed ladder and $\xi=0.92$ for the open one. These results show that the rung configurations are more important for the closed ladder than for the open one, which is in agreement with the values taken by the variational parameters. The DMRG method yields $\xi=5 \sim 10^{10}$ for open ladders, while the QMC method yields $\xi=7.1$ (closed) and $\xi=10.3$ (open). ${ }^{6}$ As expected the dimer-RVB Ansatz gives a much shorter correlation length but it reproduces the fact that $\xi_{\text {closed }}<\xi_{\text {open }}$.

We have also studied the case when the Hamiltonian (1) becomes that of two decoupled two-leg ladders, i.e., $J^{\prime \prime}$ $=J^{\prime \prime \prime}=0$. Curiously enough, our Ansatz yields a GS with bonds connecting the two ladders. The GS energy so obtained is a bit lower than the one of two uncoupled two-leg ladders and the correlation length $\xi=0.97$ is larger than in the uncoupled case $\xi=0.79 .^{9}$

\section{CONCLUSIONS}

In summary we have shown in this paper that the dimerRVB Ansatz gives a correct qualitative picture of the shortrange RVB state of the four-leg ladder. We have found interesting interference effects between resonating valence bond configurations that should probably carry over more realistic Ansätze which must include longer valence bonds. The next step is to generalize our methods to the doped four-leg ladders where one can study the phenomena of phase separation and stripe formation. ${ }^{4,15}$ Previous applications of the RVA method to the two-leg $t$ - $J$ ladder, ${ }^{16}$ the necklace $t-J$ ladder, ${ }^{17}$ and the two-leg Hubbard model, ${ }^{18}$ suggest that this goal is worth pursuing.

\section{ACKNOWLEDGMENTS}

We would like to thank J. Dukelsky for conversations and the Centro de Supercomputacion Complutense for the allocation of CPU time in the SG-Origin 2000 Parallel Computer. This work was supported by the DGES Spanish Grant No. PB97-1190 (G.S. and M.A.M.-D.).
${ }^{1}$ M. Takano, Z. Hiroi, M. Azuma, and Y. Takeda, Jpn. J. Appl. Phys., Suppl. 7, 3 (1992).

${ }^{2}$ E. Dagotto and T. M. Rice, Science 271, 618 (1996).

${ }^{3}$ S. R. White, R. M. Noack, and D. J. Scalapino, Phys. Rev. Lett. 73, 886 (1994).

${ }^{4}$ S. White and D. J. Scalapino, Phys. Rev. B 55, 14701 (1997).

${ }^{5}$ S. Gopalan, T. M. Rice, and M. Sigrist, Phys. Rev. B 49, 8901 (1994).

${ }^{6}$ M. Greven, R. J. Birgeneau, and U.-J. Wiese, Phys. Rev. Lett. 77, 1865 (1996); B. Frischmuth, B. Ammon, and M. Troyer, Phys. Rev. B 54, R3714 (1996); O. F. Syljuasen, S. Chakravarty, and M. Greven, Phys. Rev. Lett. 78, 4115 (1997).

${ }^{7}$ E. Dagotto, J. Riera, and D. J. Scalapino, Phys. Rev. B 45, 5744 (1992); Y. Nishiyama, N. Hatano, and M. Suzuki, J. Phys. Soc. Jpn. 65, 560 (1996).

${ }^{8}$ Y. Fan and M. Ma, Phys. Rev. B 37, 1820 (1988).

${ }^{9}$ G. Sierra and M. A. Martin-Delgado, Phys. Rev. B 56, 8774 (1997); For a review on the recurrent variational method (RVA), see M.A. Martin-Delgado and G. Sierra, in Density Matrix Renormalization, edited by I. Peschel et al. (Springer-Verlag, Berlin, 1999).

${ }^{10}$ S. A. Kivelson, D. S. Rokshar, and J. P. Sethna, Phys. Rev. B 35, 8865 (1987).
${ }^{11}$ M. Havilio, Phys. Rev. B 54, 11929 (1996).

${ }^{12}$ S. Liang, B. Doucot, and P. W. Anserson, Phys. Rev. Lett. 61, 365 (1988).

${ }^{13}$ The number of dimers $D_{M, N}$ on a large rectangular lattice $M$ $\times N$ is given by the formula $D_{M . N}=a^{M N}$ where $a=1.3385$ [P.W. Kasteleyn, Physica 27, 1209 (1961)]. In a $n_{l}$-ladder with $N$ rungs we find that the number of dimers is given by $F_{n_{l}, N}$ $=f_{n_{l}}^{N}$. Comparing both formulas we expect that $a_{n_{l}} \equiv f_{n_{l}}^{1 / n_{l}}$ must converge to $a$ in the large $n_{l}$ limit. Indeed we find $a_{2}$ $=1.272, a_{3}=1.245, a_{4}=1.298, a_{5}=1.288, a_{6}=1.310$. Even ladders converge more rapidly than odd ones. In the latter computation we use the RVA method with bonds of length 1 and open BC's. For the closed BC's 4-ladder we find $a_{4}=1.390$, which is a large coefficient.

${ }^{14}$ S. Ostlund and S. Rommer, Phys. Rev. Lett. 75, 3537 (1995); J. M. Roman, G. Sierra, J. Dukelsky, and M. A. Martin-Delgado, J. Phys. A 31, 9729 (1998).

${ }^{15}$ T. Tohyama C. Gazza, C. T. Shih, Y. C. Chen, T. K. Lee, S. Maehawa, and E. Dagotto, Phys. Rev. B 59, R11 649 (1999).

${ }^{16}$ G. Sierra, M. A. Martin-Delgado, J. Dukelsky, S. R. White, and D. J. Scalapino, Phys. Rev. B 57, 11666 (1998).

${ }^{17}$ G. Sierra, M. A. Martin-Delgado, S. R. White, D. J. Scalapino, and J. Dukelsky, Phys. Rev. B 59, 7973 (1999).

${ }^{18}$ E. H. Kim, G. Sierra, and D. Duffy, Phys. Rev B 60, 5169 (1999). 